$$
\begin{array}{r}
\text { Pontifícia Universidade Católica } \\
\text { do Rio de Janeiro }
\end{array}
$$

Fernando Gervasio Bastos Visser

\title{
Avaliação de Employee Stock Options com Preços de Exercício Estocásticos
}

Dissertação de Mestrado

Dissertação apresentada como requisito parcial para obtenção do grau de Mestre pelo Programa de PósGraduação em Engenharia de Produção do Departamento de Engenharia Industrial da PUC-Rio.

Orientador: Prof. Tara Keshar Nanda Baidya

Rio de Janeiro

Outubro de 2009 


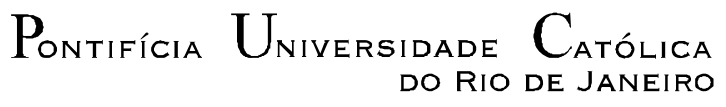

\section{Fernando Gervasio Bastos Visser}

\section{Avaliação de Employee Stock Options com Preços de Exercício Estocásticos}

Dissertação apresentada como requisito parcial para obtenção do grau de Mestre pelo Programa de PósGraduação em Engenharia de Produção da PUC-Rio. Aprovada pela Comissão Examinadora abaixo assinada.

Prof. Tara Keshar Nanda Baidya Orientador Departamento de Engenharia Industrial - PUC-Rio

Prof. Carlos Patrício Samanez Departamento de Engenharia Industrial - PUC-Rio

Prof. Fernando Antônio Lucena Aiube Departamento de Engenharia Industrial - PUC-Rio

Prof. Katia Maria Carlos Rocha Departamento de Engenharia Industrial - PUC-Rio

Dr. Luiz Felipe Pinheiro de Andrade Itaú Unibanco - Holding/SP

Prof. José Eugenio Leal Coordenador Setorial do Centro Técnico Científico - PUC-Rio 
Todos os direitos reservados. É proibida a reprodução total ou parcial do trabalho sem autorização da universidade, do autor e do orientador.

\section{Fernando Gervasio Bastos Visser}

Economista formado pela Escola de Pós Graduação em Economia (EPGE) da Fundação Getulio Vargas (FGV-Rio) em dezembro de 2005.

Ficha Catalográfica

Visser, Fernando Gervasio Bastos

Avaliação de employee stock options com preços de exercício estocásticos / Fernando Gervasio Bastos Visser ; orientador: Tara Keshar Nanda Baidya. - 2009.

135 f. : il. (color.) ; $30 \mathrm{~cm}$

Dissertação (Mestrado em Engenharia Industrial) - Pontifícia Universidade Católica do Rio de Janeiro, Rio de Janeiro, 2009.

Inclui bibliografia

1. Engenharia industrial - Teses. 2. Employee stock options. 3. Planos de opções. 4. Opções financeiras. 5. Opções de barreira. 6. Derivativos. 7. Finanças. 8. Processos estocásticos. I. Baidya, Tara Keshar Nanda. II. Pontifícia Universidade Católica do Rio de Janeiro. Departamento de Engenharia Industrial. III. Título.

CDD: 658.5 


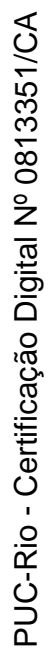

Dedico à minha família, que sempre me incentivou e me apoiou. 


\section{Agradecimentos}

Agradeço à minha família por todo o apoio e compreensão ao longo desta trajetória. Em especial à minha mãe Sandra e ao meu pai Koenraad por tudo o que sempre fizeram e continuam fazendo por mim. Ao meu irmão Ricardo pelo companheirismo.

À Thais, pela compreensão e parceria sempre.

Aos funcionários do DEI, muito obrigado por toda a ajuda.

Aos professores, agradeço pela ajuda e orientação prestada. Em particular ao professor Tara Baidya pela imensa colaboração.

Aos meus amigos de turma, sempre dispostos a auxiliar e a discutir questões conceituais. Em especial à Úrsula, Rodrigo, Fernando e Frances muito obrigado pela ajuda.

Susana Furquim, pela imensa ajuda na discussão de pontos técnicos e pela leitura atenciosa do texto.

À Coordenação de Aperfeiçoamento de Pessoal de Nível Superior (CAPES) pela ajuda financeira. 


\section{Resumo}

Visser, Fernando Gervasio Bastos; Baidya, Tara Keshar Nanda (Orientador). Avaliação de employee stock options com preços de exercício estocásticos. Rio de Janeiro, 2009. 135p. Dissertação de Mestrado

- Departamento de Engenharia Industrial, Pontifícia Universidade Católica do Rio de Janeiro.

As employee stock options ("ESOs") podem ser consideradas um dos instrumentos de remuneração e retenção mais importantes do mundo corporativo. Contudo, a crise financeira internacional desencadeada em 2008 despertou a atenção da sociedade para antigas práticas das empresas. Em particular a discussão a respeito dos pacotes de remuneração dos executivos tem ganhado cada vez mais importância. Enquanto muitos defendem que as ESOs forneceram incentivos à tomada irresponsável de decisões por parte dos executivos das grandes corporações, o presente trabalho tomou a crise mundial como motivador para apresentar uma modalidade de opção ainda pouco utilizada: a ESO com preço de exercício atrelado a um índice. Ainda que seu valor seja menor que o de uma opção tradicional, seu desenho fornece incentivos mais poderosos à tomada de decisões que visem à maximização de valor para o acionista. Neste sentido, ESOs indexadas figuram como uma interessante possibilidade na resolução do problema entre principal e agente, neste caso representado pelos acionistas e executivos, respectivamente. O presente trabalho apresenta e desenvolve modelos de apreçamento para ESOs indexadas em linha com as diretrizes gerais definidas pelos padrões contábeis nacionais e internacionais, tais como a política de exercício antecipado e o cancelamento de opções. O objetivo é, portanto servir como motivador para a utilização de modelos de apreçamento mais precisos por parte das empresas.

\section{Palavras-chave}

Employee stock options; planos de opções; opções financeiras; opções de barreira; derivativos; finanças; processos estocásticos. 


\section{Abstract}

Visser, Fernando Gervasio Bastos; Baidya, Tara Keshar Nanda (Advisor). Valuation of employee stock options with stochastic exercise prices. Rio de Janeiro, 2009. 135p. MSc. Dissertation - Departamento de Engenharia Industrial, Pontifícia Universidade Católica do Rio de Janeiro.

Employee stock options ("ESOs") can be considered one of the most important compensation and retention instruments of the corporate world. The credit crunch crisis of 2008, though, has drawn society's attention towards certain practices of corporations. In particular, the debate over the compensation packages granted to executives has gained importance. While many stand that ESOs have given incentives to the irresponsible decisions made by large corporation executives, this dissertation takes the economic crisis as a motivator and presents an option that is still barely used: an ESO with an exercise price that follows an index. Even though the value of an indexed ESO is less than the value obtained by a traditional option, its design provides stronger incentives to decisions that maximize shareholder value. In this sense, indexed ESOs appear as an interesting alternative in solving the principal-agent problem, in this case represented by shareholders and executives, respectively. This dissertation presents and develops option pricing models for indexed ESOs that are acceptable under the general guidelines defined by national and international accounting standards; such as premature exercise and option forfeiture. The objective is therefore to motivate corporations in the adoption of more adequate pricing models.

\section{Keywords}

Employee stock options; compensation packages; financial options; barrier options; derivatives; finance; stochastic processes. 


\section{Sumário}

1. Introdução 15

2. Caracterização das ESOs 19

2.1 Problema da agência 19

2.2 Principais características das ESOs 20

2.3 Implicações: valor versus custo 22

2.3.1 Definições 23

2.3.2 Por que ESOs são usadas por empresas? 23

2.3.3 Amenizando o problema 24

2.3.4 Exercício antecipado 25

2.4 Breve histórico 26

2.4.1 EUA 26

2.4.2 Brasil 28

2.4.2.1 Análise setorial 29

2.4.2.2 Política de remuneração agressiva 33

2.4.2.3 Perfil dos planos de opções no Brasil 34

2.4.2.4 Modelos de apreçamento 35

3. Contabilização 36

3.1 Valor justo de um ativo (fair market value) 36

3.2 Afinal: ESOs são ou não despesa? 37

3.2.1 Argumentos contra e a favor da despesa 38

3.3 Evolução das Normas Contábeis 40

3.3.1 Estados Unidos 40

3.3.1.1 Accounting Principles Board $25 \quad 40$

3.3.1.2 SFAS $123 \quad 41$

3.3.1.3 SFAS 123 Revisado 42

3.3.2 Europa: IFRS 2 42

3.3.3 Brasil: Pronunciamento Técnico CPC 10

3.3.3.1 Modelos de apreçamento 44

4. Revisão bibliográfica 45

4.1 Modelos baseados em maximização de utilidade esperada $\quad 45$

4.2 Modelos baseados em maximização do payoff esperado 47

4.3 Modelos para apreçamento de ESOs indexadas 49

4.4 Literatura de ESOs no Brasil 51

5. Apreçamento de ESOs com preço de exercício fixo 53

5.1 Modelo de Black e Scholes (1973) e Merton (1973) 53

5.2 Modelo de Cox, Ross e Rubinstein (1979) para opção

5.3 Modelo de Hull e White (2004) 57

6. Apreçamento de ESOs com preço de exercício estocástico $\quad 59$

6.1 Retorno absoluto versus relativo 59 
6.2 Opções Indexadas 61

6.2.1 Prós e Contras 62

6.2.2 Metodologia de apreçamento - Margrabe (1978) 63

6.2.2.1 Formulação da equação diferencial parcial 64

6.2.2.2 Solução encontrada 67

6.2.3 ESO indexada européia - Johnson e Tian (2000) 68

6.2.4 ESO indexada americana - Modelo binomial indexado 72

6.2.4.1 Algoritmo do modelo binomial indexado 76

6.2.5 Modelo binomial indexado estendido 77

6.2.5.1 Política de exercício $\quad 77$

6.2.5.2 Algoritmo do modelo binomial indexado estendido 78

6.2.6 Estimação de parâmetros $\quad 80$

6.2.6.1 Coeficiente de correlação $(\rho) \quad 80$

6.2.6.2 Taxa de cancelamentos $(\omega) \quad 80$

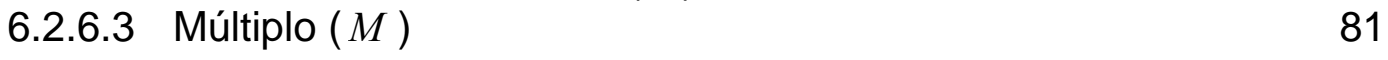

7. Resultados $\quad 82$

7.1 Validação do modelo para opções européias 82

7.1.1 Johnson-Tian (2000) 83

7.1.2 Simulação de Monte Carlo 83

7.1.2.1 Decomposição de Cholesky 84

7.1.2.2 Equações de simulação

$\begin{array}{lll}\text { 7.1.2.3 Resultados } & 85\end{array}$

$\begin{array}{lll}\text { 7.1.3 Modelo binomial indexado } & 88\end{array}$

7.2 Validação do modelo para opções americanas 90

$\begin{array}{lll}7.2 .1 & \text { Caso base } & 90\end{array}$

7.2.1.1 Opções européias 91

$\begin{array}{lll}\text { 7.2.1.2 } & \text { Resultados } & 91\end{array}$

7.2.1.3 Convergência $\quad 92$

7.2.1.4 Valor no tempo das opções 93

\begin{tabular}{ll} 
7.2.2 & Curva de gatilho \\
\hline
\end{tabular}

7.2.2.1 Políticas de exercício 95

7.2.3 Análise de sensibilidade nos parâmetros 96

$\begin{array}{lll}\text { 7.2.3.1 Múltiplo }(M) & 97\end{array}$

7.2.3.2 Taxa de cancelamentos $(\omega) \quad 99$

7.2.3.3 Período de carência $(v) \quad 100$

$\begin{array}{lll}\text { 7.2.3.4 Correlação }(\rho) & 101\end{array}$

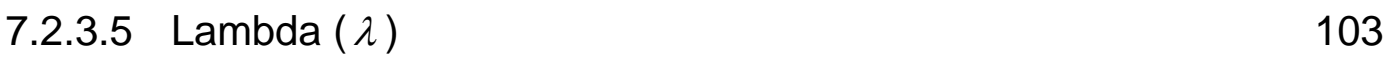

$\begin{array}{lll}7.2 .4 & \text { Incentivos } & 105\end{array}$

$\begin{array}{ll}\text { 8. Estudo de caso } & 107\end{array}$

$\begin{array}{ll}8.1 \text { Visão geral } & 107\end{array}$

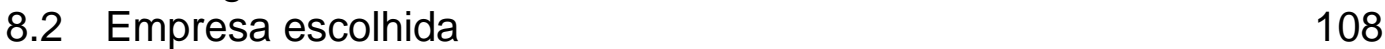

$\begin{array}{ll}8.3 \text { Cálculos efetuados } & 109\end{array}$

8.4 Análise dos resultados 111

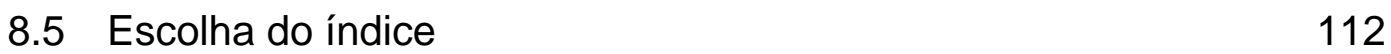

9. Conclusão 115 
10. Anexo

10.1 Definição dos processos estocásticos 118

10.2 Excesso de retorno 119

10.3 Processo estocástico do derivativo (F) 119

10.4 Definição do processo estocástico do preço de exercício 121

10.5 Volatilidade total 123

10.6 Modelo binomial para ESO indexada americana 123

10.7 Modelo binomial para ESO indexada com múltiplo de exercício (modelo estendido) 127

10.8 Demonstração das equações da simulação de Monte Carlo 127

$\begin{array}{lll}\text { 10.8.1 Simulação do preço da ação } & 127\end{array}$

10.8.2 Simulação do ativo referência 128

11. Referências bibliográficas 130 


\section{Lista de tabelas}

Tabela 1- Modelos usados - 31/12/2008 35

Tabela 2 - Parâmetros do caso base - opção européia 82

Tabela 3 - Valores calculados 83

Tabela 4 - Solução analítica 83

Tabela 5 - Convergência do método de Monte Carlo 86

Tabela 6 - Evolução da variância 86

Tabela 7 - Resultados do modelo binomial indexado 88

Tabela 8 - Comparação dos modelos 88

Tabela 9 - Parâmetros adicionais 90

Tabela 10 - Comparação dos modelos (americanas) 91

Tabela 11 - Preços de exercício 104

Tabela 12 - Principais características dos planos de opções indexados no Brasil em 31/12/2008 107

Tabela 13 - Principais características do plano 109

Tabela 14 - Parâmetros calculados (IGPM) 110

Tabela 15 - Resultado obtido com o modelo binomial indexado para o IGPM (valores em R\$) 110

Tabela 16 - Diferença entre modelos (IGPM) 111

Tabela 17 - Parâmetros calculados (Ibovespa) 113

Tabela 18 - Resultado obtido com o modelo binomial indexado Ibovespa (valores em R\$) 113

Tabela 19 - Diferença entre modelos (Ibovespa) 114

Tabela 20 - Valor de exercício das opções em diferentes cenários $\quad 115$ 


\section{Lista de figuras}

Figura 1 - Despesa média com ESOs como percentual do lucro para empresas do S\&P500

Figura 2 - Proporção de ESOs nas recentes aberturas de capital da Bovespa

Figura 3 - Composição setorial das empresas com ESOs no Ibovespa 29

Figura 4 - Composição setorial das empresas com ESOs nas recentes aberturas de capital

Figura 5 - Setores que proporcionalmente mais utilizam ESOs no Ibovespa

Figura 6 - Setores que proporcionalmente mais utilizam ESOs nas recentes aberturas de capital

Figura 7 - Despesa com ESOs como percentual do lucro líquido de 2008 34

Figura 8 - Trajetórias possíveis para a ação 55

Figura 9 - Distribuições do modelo binomial 56

Figura 10 - Dinâmica do preço da ação (S) e do ativo referência (H) 73

Figura 11 - Dinâmica do numerário 73

Figura 12 - Caminho possível para $\mathrm{S}$ e $\mathrm{H}$ 85

Figura 13 - Histograma dos payoffs no vencimento 87

Figura 14 - Histograma obtido na simulação para o valor da opção $\quad 87$

Figura 15 - Convergência dos métodos utilizados. 89

Figura 16 - Convergência do modelo binomial indexado estendido $\quad 92$

Figura 17 - Valor da opção para diferentes preços da ação (caso base) 93

Figura 18 - Curva de gatilho normalizado para o modelo binomial indexado (carência $=5$ anos)

Figura 19 - Curva de gatilho normalizado para o modelo binomial indexado (carência = zero)

Figura 20 - Comparação das políticas de exercício 96

Figura 21 - Convergência da opção com $M(\omega=0 \%) \quad 97$

Figura 22 - Convergência da opção com M (caso base, $\omega=1,5 \%) \quad 98$ 
Figura 23 - Convergência para diferentes valores de $M$

Figura 24 - Efeito do aumento da taxa de cancelamentos no valor das ESOs

Figura 25 - Efeito da carência no valor das ESOs 101

Figura 26 - Efeito da correlação entre a ação e o índice 102

Figura 27 - Volatilidade total como função da volatilidade do índice 103

Figura 28 - Valor da opção para diferentes lambdas 104

Figura 29 - Delta hedge dos modelos 106 


\section{Lista de abreviaturas}

ALL - América Latina Logística

ANEEL - Agência Nacional de Energia Elétrica

APB - Accounting Principles Board

$\mathrm{BI}$ - Binomial indexado

BIE - Binomial indexado estendido

Bovespa - Bolsa de Valores de São Paulo

BSM - Black, Scholes e Merton

CAPM - Capital Assets Pricing Model

CPC - Comitê de Pronunciamentos Contábeis

CVM - Comissão de Valores Mobiliários

ESO - Employee stock option

ESOARS - Employee Stock Option Appreciation Rights Securities

FASB - Financial Accounting Standards Board

HW - Hull e White

IASB - International Accounting Standards Board

Ibovespa - Índice da Bolsa de Valores de São Paulo

IFRS - International Financial Reporting Standards

IGP-M - Índice Geral de Preços de Mercado

ISO - Incentive stock option

JT - Johnson e Tian

MAR - Margrabe

MC - Monte Carlo

ROA - Retorno sobre os ativos

S\&P 500 - Standard \& Poor's 500

SEC - Securitires and Exchange Comission

SFAS - Statement of Financial Accounting Standards

SPE - Sociedade de Propósito Específico 